\title{
Glycine Receptors are Present in Human Epidermis
}

\author{
Dirk Booken, Carmen Henrich-Kellner, Diana Klein, Sergij Goerdt and Hjalmar Kurzen*
}

\author{
Department of Dermatology, Venerology and Allergology, Medical Faculty of Mannheim, University of Heidelberg, \\ Germany
}

\begin{abstract}
The inhibitory glycine receptor (GlyR) is a member of the nicotinoid receptor superfamily. This heteropentameric $\mathrm{Cl}^{-}$channel is composed of different $\alpha(1-4)$ and a $\beta$-subunit and mediates fast synaptic transmission in the central nervous system. Since glycine, the natural ligand of GlyR has been found to enhance epidermal barrier recovery; we aimed at characterizing GlyR distribution in human skin and their function in skin physiology. We detected different $\alpha$ subunits and the $\beta$ - GlyR subunit on mRNA and protein level in human skin and cultured keratinocytes and fibroblasts. In cultured human keratinocytes but not in fibroblasts, glycine induced proliferation. Epidermis-equivalents were significantly thicker than control if cultured in the presence of glycine. In human skin, GlyR immunoreactivity (IR) was detected in the upper epidermal layers. In eczema and psoriasis, GlyR IR was reduced in areas with parakeratosis suggesting a role of GlyR in terminal differentiation and epidermal barrier control.
\end{abstract}

Keywords: Epidermal barrier, chloride, strychnine, amino acid, skin.

\section{INTROCUCTION}

Structurally, glycine (aminoethanoic acid, $\mathrm{C}_{2} \mathrm{H}_{5} \mathrm{NO}_{2}$ ) is the simplest of the 20 standard proteinogenic amino acids, and yet it has a number of important and distinct functions in the body [1]. In addition to its function as part of peptides and proteins, glycine is a major inhibitory neurotransmitter in adult spinal cord and brain stem, where it controls both motor and sensory pathways. After its release from the presynaptic terminals of glycinergic interneurons, glycine exerts multiple functions in the central nervous system, as an inhibitory neurotransmitter through activation of specific, $\mathrm{Cl}^{-}-$ permeable, ligand-gated ionotropic receptors (GlyR) and as an obligatory co-agonist with glutamate on the activation of N-methyl-D-aspartate (NMDA) receptors [2, 3]. The hyperpolarizing action of glycine is selectively antagonized by strychnine, the most potent GlyR antagonist known [4].

The GlyR of different species are composed of two glycosylated integral membrane proteins, the $\alpha 1-\alpha 4$ - subunit of $48 \mathrm{kDa}$ and the $\beta$ - subunit of $58 \mathrm{kDa}$. In addition, in neuronal tissues, there is an associated peripheral membrane protein of $93 \mathrm{kDa}$ named gephyrin [5-7]. The primary structures of GlyR $\alpha$ - and $\beta$ - subunits deduced by molecular cloning show significant sequence and structural similarity to nicotinic acetylcholine receptor (nAChR), $\gamma$-aminobutyric acid type A (GABA A) receptor, and serotonin type 3 (5HT3) receptor proteins. In situ hybridization showed that the different GlyR subunit genes exhibit unique spatial and temporal expression patterns in spinal cord, brain stem, and some higher brain regions. GlyR $\alpha 2$ - subunit transcripts predominate in the embryonic and neonatal brain and spinal cord, but are replaced

*Address correspondence to this author at the Department of Dermatology, Venereology and Allergology, Medical Faculty of Mannheim, RuprechtKarls University of Heidelberg, Theodor-Kutzer-Ufer 1-3, D-68167 Mannheim, Germany; Tel: 0049 (0) 621 383-2280; Fax: 0049 (0) 621 383-3815; E-mail: Hjalmar.Kurzen@haut.ma.uni-heidelberg.de or

Hjalmar.Kurzen@nexgo.de postnatally by the $\alpha 1$ - or $\alpha 3$ - subunit mRNAs. Notably, transcripts for the GlyR $\beta$ - subunit are widely expressed even in brain regions that lack $\alpha 1-, \alpha 2-$, and $\alpha 3-$ mRNAs [4].

Recently, several unexpected properties of glycine have been discovered. Like the structurally related nicotinic acetylcholine receptors [8], GlyR were shown to be expressed in peripheral, non-neuronal tissues and cells like macrophages and leukocytes suggesting immune-modulatory functions $[9$, $10]$.

Glycine is widely used in cosmetic products because of a suggested beneficial effect on skin collagen synthesis ("skin conditioning" according to INCI). Since glycine has no side chain, it can fit into many places where no other amino acid can. While most proteins contain only small quantities of glycine, collagen is composed of about one-third glycine [11]. Hair follicle and hair cortex cells have been shown to contain relatively high amounts of glycine- and tyrosine-rich proteins [12]. In a mouse model, topical applications of glycine, effectively inhibited the wrinkle formation induced by repeated tape stripping of chronic eczematous dermatitis. Furthermore, glycine is an effective inducer of epidermal barrier recovery after tape stripping [13], an effect antagonized by strychnine. In addition, glycine-induced influx of chloride ions into epidermal keratinocytes regulates exocytosis of epidermal lamellar bodies and skin permeability barrier homeostasis [14].

Since the presence of GlyR in the skin has not been reported before, we wanted to know, whether the observed in vivo effects were attributable to specific, functional active GlyR in the epidermis.

\section{MATERIAL AND METHODS}

\section{Tissues}

Normal human adult skin from the margins of routine surgical procedures and skin biopsies from patients with psoriasis or eczema were obtained after informed written consent according to the declaration of Helsinki and approved by the local ethical committee. Samples were frozen in 
isopentane cooled in liquid nitrogen immediately after removal and stored at $-80^{\circ} \mathrm{C}$. Fresh samples were used to isolate normal epidermal keratinocytes and human dermal fibroblasts.

\section{Cell Culture}

Human dermal fibroblasts (HDF) and normal epidermal keratinocytes (NEK) were isolated from skin specimens as described [15]. HDF were grown in DMEM (Dulbecco's modified Eagle's medium, Bio Whittacker, Verviers, Belgium) containing $0.4 \mathrm{mM}$ glycine, supplemented with $10 \%$ fetal calf serum (FCS). For organotypic cocultures (OTC) mitomycin C $(8 \mu \mathrm{g} / \mathrm{ml}$; Sigma, Steinheim, Germany) treated HDF were used. NEK were grown on feeder HDF in FAD medium (DMEM: Ham's F12 / 3:1) with 100 U/ml penicillin, $100 \mu \mathrm{g} / \mathrm{ml}$ streptomycin and supplemented with $5 \% \mathrm{FCS}, 5 \mu \mathrm{g} / \mathrm{ml}$ insulin, $1 \mathrm{ng} / \mathrm{ml}$ recombinant EGF, $10^{-10} \mathrm{M}$ cholera toxin, $10^{-4} \mathrm{M}$ adenine and $0.4 \mu \mathrm{g} / \mathrm{ml}$ hydrocortisone (Sigma) as described [16]. FAD medium contains $0.325 \mathrm{mM}$ glycine.

\section{Proliferation Assay}

On day one, cells were seeded on a 96well- microtiter plate at 4000 cells per well. Cells were grown for three days in the presence of different final concentrations of glycine (up to $10 \mathrm{mM}$ ), glycine and strychnine (each up to $10 \mathrm{mM}$ ) or strychnine alone (up to $3 \mathrm{mM}$, both from Sigma) or in basal culture medium alone. The medium was changed every day, in order to exclude degradation artefacts. On day 3 cells were washed with HEPES buffer, fixed with $2 \%$ buffered formaldehyde, stained with $1 \%$ methylene blue and air dried. After thorough washing, methylene blue concentration in the extraction buffer $(0.1 \mathrm{~N} \mathrm{HCL})$ was determined using an ELISA reader at $620 \mathrm{~nm}$. Methylene blue is incorporated into the DNA in a linear proportion to the cell number. After normalization to a standard curve, relative cell density could be assessed [17]. All proliferation analyses were performed in 2-times quadruplicate manner for each condition and repeated twice. Statistical analysis was performed using a standard unpaired student's t-test, available on statpages.net.

\section{Organotypic Cocultures (OTC)}

As described in detail before (15) lyophilized collagen type I was resolubilised at $4 \mathrm{mg} / \mathrm{ml}$ in $0.1 \%$ acetic acid and kept at $4{ }^{\circ} \mathrm{C}$. Calf skin collagen I was obtained from IBFB (Leipzig, Germany). The ice-cold collagen solution was mixed with Hanks salt with phenol red (Seromed Biochrom $\mathrm{KG}$, Berlin, Germany) and adjusted to $\mathrm{pH}$ 7.4. The HDF were resuspended in FCS and added to the gel to give $10^{5}$ cells $/ \mathrm{ml}$ in the collagen gel under cautious stirring. $2.5 \mathrm{ml}$ of the collagen / HDF solution was poured onto filter inserts ( $3 \mu \mathrm{m}$ pore size, Falcon) of a 6-fold deep well plate (BioCoat, Becton-Dickinson, Heidelberg. Germany). After gelation, glass rings were mounted on the gels in order to define the area of epithelial cell growth. The gels were equilibrated by complete immersion in culture medium for 24-48h. Thereafter, the NEK were plated inside the glass rings at a density of $10^{6}$ cells per well. After 2 days the glass rings were carefully removed and the medium level was lowered to the lower part of the gel, thus raising the keratinocytes to the air-liquid interface and restricting nourishment to diffusion from below. The air lift procedure is defined as day 0 of OTC. OTCs were grown in FAD-Medium with $10 \%$ FCS and $50 \mu \mathrm{M}$ ascorbic acid. Medium was changed every 2-3 days. At the end of the experiment, the epidermal-collagen specimens were snap frozen in liquid nitrogen and stored at $-80^{\circ} \mathrm{C}$.

In a single experiment, OTCs of one condition were always performed twice. All cell culture experiments were repeated at least twice. Glycine and strychnine were added to the OTCs on the day of the air-lift to reach final in concentrations ranging from $10 \mu \mathrm{M}$ to $10 \mathrm{mM}$.

\section{Determination of Epithelial Thickness}

The epithelial thickness obtained using the described culture conditions was determined by measuring the thickness of the malpighian layer at 2 representative points of each specimen, so that for each condition, at least 6 different values were obtained. Statistical analysis was performed using an unpaired student's t-test available on www.statpages.net.

\section{Immunofluorescence Staining}

For immunofluorescence analysis, $5 \mu \mathrm{m}$ cryostat sections of human scalp skin were either used air dried and unfixed or fixed either in ice-cold acetone. Immunofluorescence reactions were performed at least 3 times on different specimens according to standard procedures [15]. After blocking unspecific binding sites with 3\% BSA in Tris-buffered saline (TBS), a streptavidin blocking solution and a biotin blocking solution (both Dianova, Hamburg, Germany) were applied for 15 minutes each, followed by washing in TBS. The primary antibody mab 4a (article no. 146011, Synaptic Systems, Göttingen, Germany) was applied to the specimens for $1 \mathrm{~h}$ at room temperature (dilution 1:100). Biotin-coupled secondary antibodies were applied for one hour at room temperature: preabsorbed goat anti-mouse antiserum, obtained from Dianova. Antibody binding was visualized with $\mathrm{Cy}-3$ coupled streptavidin (Dianova). Negative controls demonstrating the specificity of obtained signals were performed by replacing the primary antibody with PBS or an irrelevant, species - matched antibody. DAPI (Roche Diagnostics $\mathrm{GmbH}$, Mannheim, Germany) used for counterstaining the cell nuclei was added in the final incubation period at a dilution of 1: 2000. Washes ( $3 \times 10 \mathrm{~min})$ were performed after each step in PBS or TBST. Pictures were generated using a Leitz DMRB immunofluorescence microscope.

\section{Reverse Transcriptase-Polymerase Chain Reaction (RT- PCR)}

RNA was extracted from split skin (depth $2 \mathrm{~mm}$ ) using the RNeasy ${ }^{\circledR}$ fibrous tissue Kit according to the manufacturer's instructions (QIAGEN, Hilden, Germany). cDNA was synthesized from freshly isolated mRNA after DNase treatment using Superscript II (Life Technologies, Eggenstein, Germany). For PCR analyses 100ng of the respective cDNA and 20 pmol primers were used per $25 \mu \mathrm{l}$ reaction tube. Primer sequences are listed in table 1. Hot start PCR was performed using AmpliTaq Gold ${ }^{\circledR}$ (Applied Biosystems, Weiterstadt, Germany) according to standard protocols on a thermal cycler (DNA Engine DYAD ${ }^{\mathrm{TM}}$, BioRad GmbH, München, Germany). The annealing temperature was optimized for all primer pairs and ranged from $55^{\circ} \mathrm{C}$ to $60^{\circ} \mathrm{C}$. PCR products were visualized on standard $2 \%$ agarose gels with ethidium bromide. All PCR experiments were per- 
formed at least twice. Contaminating genomic DNA was excluded in all experiments using PDH (Acc.\#: XM_113395) primers that produce fragments of $102 \mathrm{bp}$ in the presence of cDNA and fragments of $200 \mathrm{bp}$ in presence of genomic DNA (not shown).

\section{Western Blot Analysis of Detergent Soluble and Insoluble Cytoplasmic Fractions}

Whole cell lysates were generated by homogenising human scalp skin in ice-cold lysis buffer $(150 \mathrm{mM} \mathrm{NaCl}, 1 \%$ NP40, $\quad 0.5 \%$ sodium-desoxycholate, $\quad 0.1 \%$ sodiumdodecylsulfate (SDS), $50 \mathrm{mM}$ Tris/HCL pH 8, supplemented with a complete Protease Inhibitor Cocktail (Roche, Mannheim, Germany) and performing 2-3 freeze-thaw cycles. Resulting pellets (membrane fraction) were solubilized in Laemmli Buffer. Protein content was determined by a detergent compatible assay (DC-Assay; BioRad). Protein samples (100 $\mu \mathrm{g}$ of total protein) were separated on $10 \%$ polyacrylamide gels and transferred to nitrocellulose membranes (BioRad, Munich, Germany), for $3 \mathrm{hrs}$ at $0.25 \mathrm{~mA} / \mathrm{cm}^{2}$ in a wetblot electrophoretic transfer cell as recommended by the manufacturer (BioRad). The membranes were rinsed and nonspecific protein binding sites were blocked by $30 \mathrm{~min}$ incubation with 5\% skim milk/ phosphate-buffered saline (PBS). Membranes were incubated with a monoclonal antiGlyR antibody (dilution 1:500, mab 4a, Synaptic Systems) overnight at $4^{\circ} \mathrm{C}$ in blocking solution. After incubation with a secondary peroxidase-conjugated anti-mouse antibody (1/10 000; Jackson IR Laboratories, West Grove, PA, USA), signals were detected with the enhanced chemiluminescence system from Amersham.

\section{RESULTS}

\section{Presence of Glycine Receptors in Human Skin}

We screened samples of mRNA from human skin of various body sites for the presence of GlyR. Using RT-PCR we obtained bands of expected sizes corresponding to mRNA of the GlyR $\alpha 2, \alpha 3$ and the $\beta$-subunit. In order to find out which cells might be responsible for the obtained positive signal, we analyzed cultured keratinocytes and fibroblasts of different donors and again, we obtained bands of expected sizes for the GlyR $\alpha 2, \alpha 3$ and the $\beta$-subunit. In addition, we found the $\alpha 1$-subunit expressed in cultured keratinocytes and fibroblasts, while in preparations of human skin, this subunit could not be detected. All subunits could be detected in a neuroblastoma cell line used as positive control (Fig. 1a).

Using a commercially available monoclonal antibody recognizing all GlyR subunits, we found bands of 48 and 58 $\mathrm{kDa}$ in immunoblots of human scalp skin total protein preparations, corresponding to the different $\alpha$ - subunits that share the same molecular weight of $48 \mathrm{kDa}$ and the $\beta$ - subunit $(58 \mathrm{kDa})$ (Fig. 1b).

\section{Localization of GlyR in Human Skin}

After demonstrating the presence of GlyR in human skin we investigated the localisation of the receptors within the skin. Using immunofluorescence of normal scalp skin, we found a bright labeling of the GlyR antibody in the upper stratum spinosum and in the stratum granulosum that could also be detected in the upper layers of hair follicle infundibu- lum (Fig. 2a,c). In addition we noticed a bright labeling of the sebaceous duct and the cuticle of inner root sheath (Fig. $\mathbf{2 c , d}$, arrows).

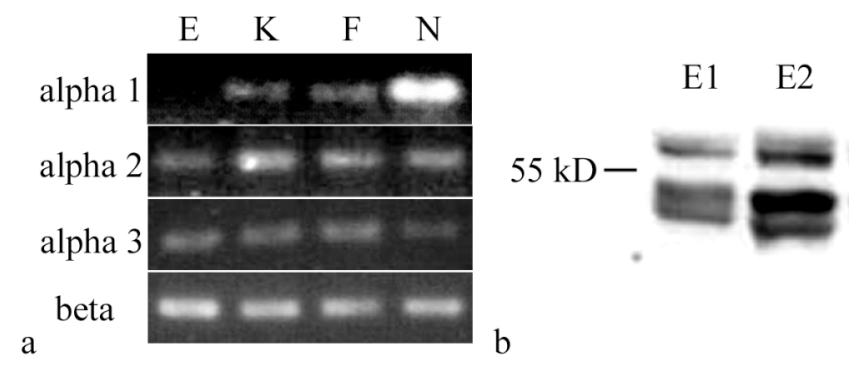

Fig. (1). GlyR expression in human skin. RT-PCR (a) and Immunoblotting (mab 4a) (b). Whole skin preparation (E), cultured keratinocytes $(\mathrm{K})$, cultured fibroblasts $(\mathrm{F})$ and in a neuroblastoma cell line $(\mathrm{N})$.

Since glycine has been reported to influence skin permeability barrier homeostasis [13, 14], we wanted to know how GlyR behave in a situation with disturbed epidermal barrier, as can be found on psoriasis or eczema $[18,19]$. Most notably, we found a striking reduction of GlyR immunoreactivity in specimens of eczema and psoriasis, restricted exactly to the parts of the epidermis covered with parakeratosis (arrow in Fig. 2b).

\section{Influence of Glycine on Keratinocyte and Fibroblast Pro- liferation}

In order to characterize the influence of glycine on basic cell functions we analysed the proliferation of keratinocytes and fibroblasts in a monolayer culture system (in the presence of glycine). After 3 days in culture, glycine displayed potent dose-dependent proliferative effects on keratinocytes with $10 \mathrm{mM}$ glycine inducing an increase in cell number to $152 \%$ of control $(\mathrm{p}<0,0001)$ while $1 \mathrm{mM}(134 \%, \mathrm{p}<0,0001)$ and $500 \mu \mathrm{M}(120 \%, \mathrm{p}=0,01)$ glycine were slightly less efficient. In contrast, fibroblast proliferation was only slightly but significantly reduced to approximately $80 \%$ of control $(p<0,05)$. The effect of glycine on keratinocyte proliferation could be abolished by addition of equimolar concentrations of the GlyR-inhibitor strychnine, while glycine-induced inhibition of fibroblast proliferation was not abolished by strychnine. Moreover, $1 \mathrm{mM}$ strychnine alone significantly inhibited keratinocyte proliferation to $82 \%$ of control $(\mathrm{p}<0,05)$ while fibroblast proliferation was not significantly altered. $100 \mu \mathrm{M}$ of strychnine had no significant effect on the numbers of both cell types (Fig. 3).

\section{Glycine Induces Epithelial Hyperplasia}

In an attempt to assess the influence of glycine on epidermal differentiation, we used an organotypical coculture system (OTC) that has been shown to produce epidermal equivalents after culture of keratinocytes at the air-liquid interface for 10-12 days. In the presence of $1 \mathrm{mM}$ glycine, we noticed a significant thickening of epithelial sheets obtained to approximately $155 \%$ (corresponding to $68 \mu \mathrm{m}$ ) of control (corresponding to $44 \mu \mathrm{M}, \mathrm{p}<0,0001$ ) (Figs. 4b,5), while glycine and equimolar concentrations of strychnine led to a complete inhibition of epidermal differentiation, reducing epidermal thickness significantly to approximately $19 \%$ of control (corresponding to $8,3 \mu \mathrm{M}, \mathrm{p}<0,0001$ ) (Figs. 4c,5). 


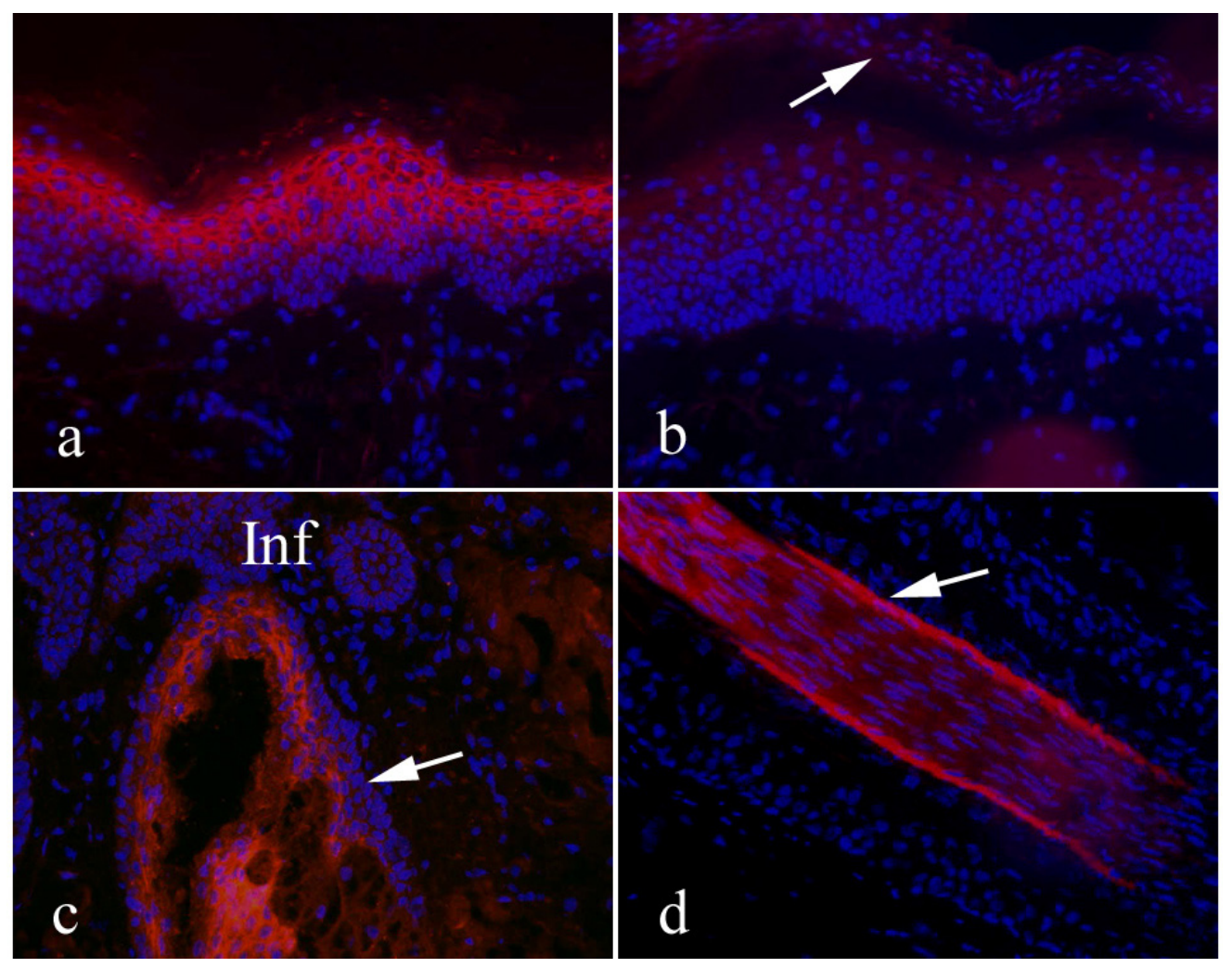

Fig. (2). Distribution of GlyR in human skin. Immunofluorescence analysis using mab 4a, counterstain of cell nuclei with DAPI. Original magnification $20 \mathrm{x}$ in a-c, $40 \mathrm{x}$ in $\mathrm{d}$.

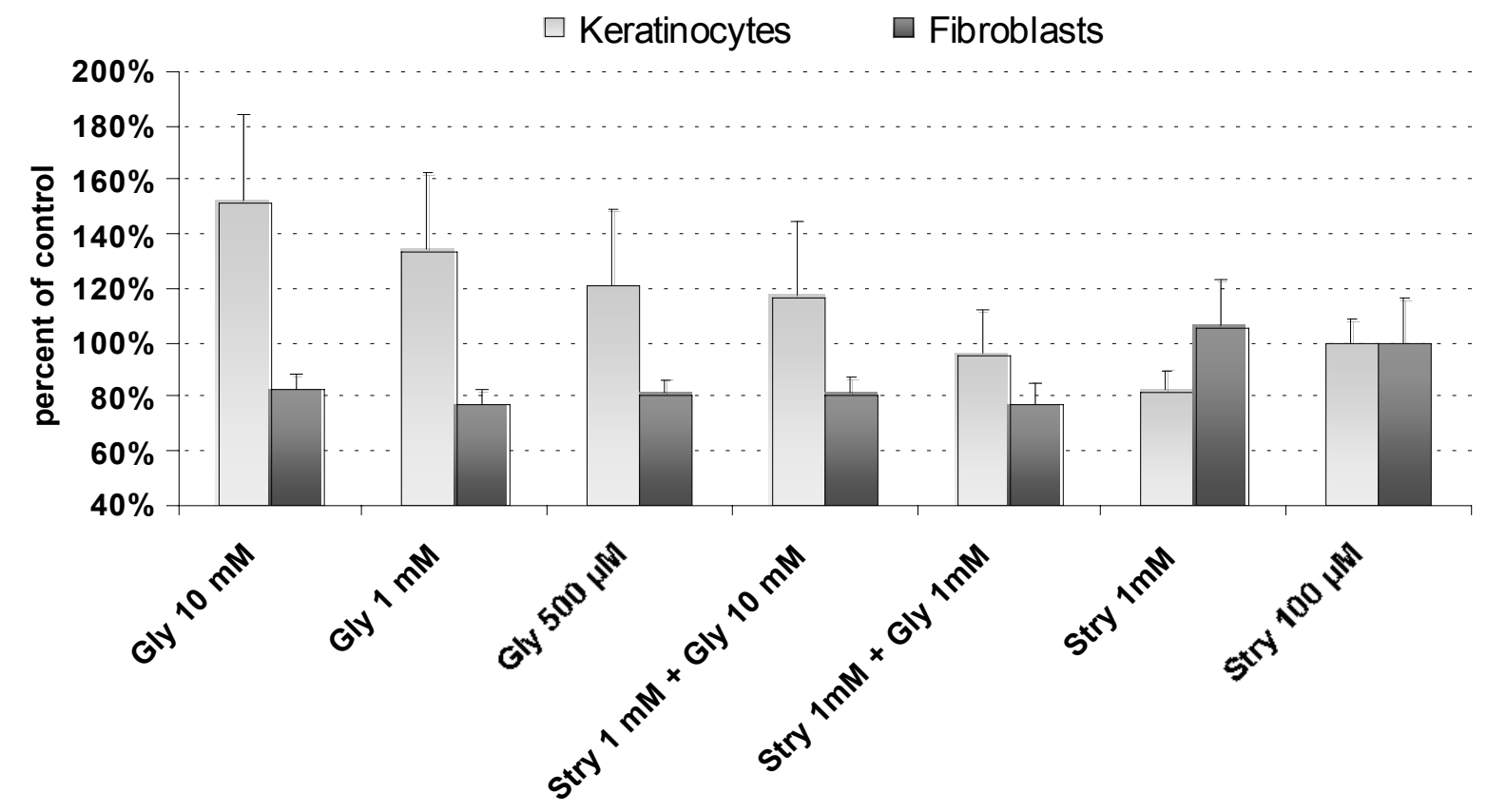

Fig. (3). Influence of glycine on keratinocyte and fibroblast proliferation in percent of control. Glycine (Gly), strychnine (Stry). ** ${ }^{*}<$ $0,0001, * p=0,01$. Bars represent standard deviation.

As we could demonstrate previously [15], strychnine alone reduced epidermal thickness to approximately $66 \%$ of control $(29 \mu \mathrm{m}, \mathrm{p}<0,001)$ (Figs. 4d,5). The difference between strychnine alone and strychnine together with glycine is not significant.

\section{DISCUSSION}

In the present study, we were able to demonstrate the presence of several GlyR subunits in human skin for the first time. The distribution of GlyR in human epidermis, as evidenced by the pan-GlyR subunit antibody used in our study, 
seems to be restricted to the upper epidermal layers that are crucially involved in the formation of a tight epidermal barrier. In addition to the presence of GlyR, as presented in this study, also gamma-aminobutyric acid (A) receptors [20], NMDA-type glutamate receptors [21], dopamine D2-like receptors [22] and acetylcholine receptors [8] are present in the epidermis and regulate barrier permeability. Apparently, GlyR in keratinocytes and fibroblasts are composed of different subunits. While the GlyR $\beta$ - subunit is present in all GlyR of other neuronal and non-neuronal tissues [4], there seems to be a differential distribution of the $\alpha$-subunits. In adults, the most common form of the native channel was shown in cross-linking studies to be a pentameric assembly of $\alpha 1$ - and $\beta$-subunits [23]. Similar to most mammalian neuroreceptors, native GlyR protein subunits are present in very low abundance in vivo [24] explaining the absence of $\alpha 1$ subunit from a human skin mRNA preparation in contrast to cultured keratinocytes and fibroblasts Remarkably, we also found GlyR $\alpha 2$ - and $\alpha 3$ - subunit mRNA in cultured fibroblasts, keratinocytes and in whole-skin preparations. Commonly, the $\alpha 2$ - subunit is found in embryonic tissues and is replaced in adult tissues by the other $\alpha$-subunits [4]. However, in adult mice, the $\alpha 2$ - subunit has been found in the retina [25] and rat macrophages and neutrophilic granulocytes [9] representing a conceivable function in adult human non-neuronal tissues. GlyR $\alpha 3$ - subunits together with $\alpha 1$ and $\beta$-subunits have been identified in several small-cell lung cancer cell lines [26]. We did not examine the GlyR $\alpha 4-$ subunit, since the complete human sequence was not available at the time of the study. In rats, the $\alpha 4$ - subunit has been found in macrophages and neutrophilic granulocytes [9].

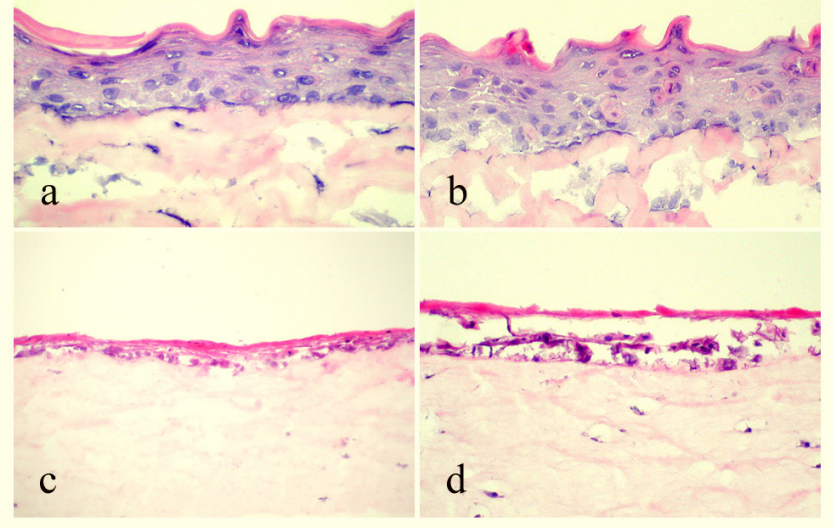

Fig. (4). Glycine induces epidermal hyperplasia in an organotypic coculture system. Control specimens (a), $1 \mathrm{mM}$ glycine (b) $1 \mathrm{mM}$ glycine and $1 \mathrm{mM}$ strychnine (c) $1 \mathrm{mM}$ strychnine alone (d) H\&E staining, Original magnification 20x.

Previous studies demonstrated the glycine-induced influx of chloride ions into epidermal keratinocytes, well in line with the structure and function of GlyR in other tissues, where GlyR were convincingly demonstrated to form ligand gated ion channels permeable for chloride $[14,24]$. We therefore conclude that at least in cultured keratinocytes, GlyR are functional and composed of different $\alpha$ and $\beta$ subunits.

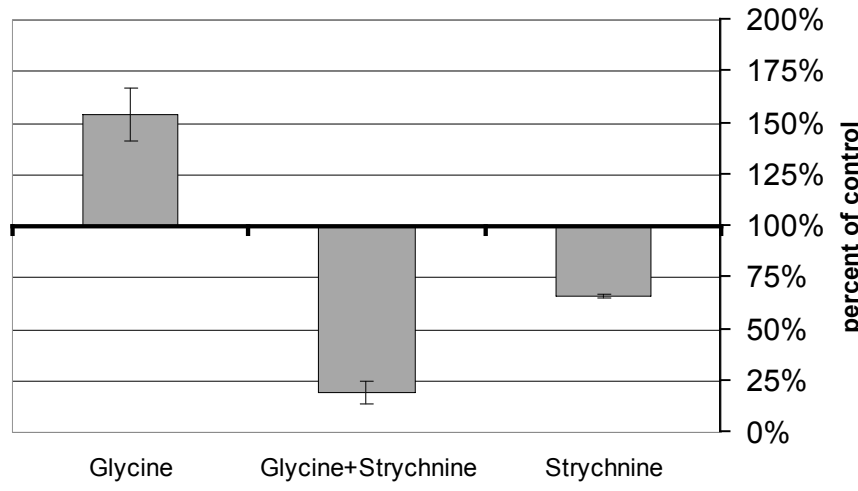

Fig. (5). Quantification of glycine impact on epithelial thickness Quantification of epidermal thickness obtained in organotypical cocultures in the presence of glycine and strychnine as shown in Fig. (4).

In order to elucidate the consequence of GlyR activation and chloride influx into keratinocytes and fibroblasts we tested basic cell functions, namely proliferation and differentiation. We could demonstrate a highly potent dose dependent activation of keratinocyte proliferation through glycine, an effect that could completely be abolished by strychnine (Fig. 3). Strychnine is a natural high affinity inhibitor of GlyR [5]. In addition it has been shown to inhibit the structurally related $\alpha 7-$ and $\alpha 9-$ nicotinic acetylcholine receptors (nAChR) present in human skin [8]. Therefore strychnine may serve as inhibitor of glycine induced effects as shown in our study. Given alone, the highly potent inhibition of $\alpha 7-$ and $\alpha 9-$ nAChR may predominate. Since either $\alpha 7$ - and $\alpha 9$ $\mathrm{nAChR}$ are present in keratinocyte and fibroblast monolayers as well as in the OTC system [15], effects observed in the presence of strychnine alone have to interpreted as nAChRinduced and not GlyR-induced (Fig. 3). Since glycineinduced inhibition of fibroblast proliferation could not be abolished by strychnine, we conclude that fibroblasts GlyR possess an additional strychnine insensitive low-affinity site with $\mathrm{Kd}$ values in the milimolar range, as has been described previously [27]. In addition, other fibroblast functions like collagen production may be influenced, which would be well in line with known effects of glycine on collagen formation [11]. Using a well characterized OTC system, we could demonstrate glycine effects on terminal differentiation and barrier formation as evidenced by an increase in epithelial thickness (Figs. 4,5). As for keratinocyte proliferation (Fig. 3), this effect could be abolished by strychnine.

Since others $[13,14]$ have suggested glycine to be an epidermal barrier recovery accelerator, we examined dermatoses with perturbed barrier function $[18,19]$. We found a significantly reduced expression of GlyR in the specimens of psoriasis or eczema with parakeratotic cornification, very much resembling the pattern found previously for the calcium-gating NMDAR1 $[28,29]$ that are activated by glutamate and glycine as an obligatory co-agonist [3].

In conclusion, our data support the findings for glycine having an important role in epidermal differentiation and specifically in epidermal barrier formation. Further studies are needed to clarify the molecular mechanisms regulating GlyR expression and the signalling cascades following chloride influx into keratinocytes. 


\section{REFERENCES}

[1] Tunnicliff G. Membrane glycine transport proteins. J Biomed Sci 2003; 10(1): 30-6

[2] Aprison MH. The discovery of the neurotransmitter role of glycine. In Glycine Neurotransmission. O.P. Ottersen \& J. StormMathiesen, Eds., John Wiley \& Sons. New York, 1990: 1-23.

[3] Aragon C, Lopez-Corcuera B. Structure, function and regulation of glycine neurotransporters. Eur J Pharmacol 2003; 479(1-3): 24962.

[4] Betz H, Kuhse J, Schmieden V, Laube B, Kirsch J, Harvey RJ. Structure and functions of inhibitory and excitatory glycine receptors. Ann NY Acad Sci 1999; 868: 667-76.

[5] Pfeiffer F, Graham D, Betz H. Purification by affinity chromatography of the glycine receptor of rat spinal cord. J Biol Chem 1982; 257(16): 9389-93.

[6] Schmitt B, Knaus P, Becker CM, Betz H. The Mr 93,000 polypeptide of the postsynaptic glycine receptor complex is a peripheral membrane protein. Biochemistry 1987; 26(3): 805-11.

[7] Prior P, Schmitt B, Grenningloh G, et al. Primary structure and alternative splice variants of gephyrin, a putative glycine receptortubulin linker protein. Neuron 1992; 8(6): 1161-70.

[8] Kurzen H, Wessler I, Kirkpatrick CJ, Kawashima K, Grando SA. The non-neuronal cholinergic system of human skin. Horm Metab Res 2007; 39(2): 125-35.

[9] Froh M, Thurman RG, Wheeler MD. Molecular evidence for a glycine-gated chloride channel in macrophages and leukocytes. Am J Physiol Gastrointest Liver Physiol 2002; 283(4): G856-63.

[10] Schilling T, Eder C. A novel physiological mechanism of glycineinduced immunomodulation: $\mathrm{Na}+$-coupled amino acid transporter currents in cultured brain macrophages. J Physiol 2004; 559(Pt 1): 35-40.

[11] Horng JC, Kotch FW, Raines RT. Is glycine a surrogate for a Damino acid in the collagen triple helix? Protein Sci 2007; 16(2): 208-15.

[12] Plowman JE, Paton LN, Bryson W. The differential expression of proteins in the cortical cells of wool and hair fibres. Exp Dermatol 2007; 16(9):707-14

[13] Matsunaga Y, Ogura Y, Ehama R, Amano S, Nishiyama T, Tagami H. Establishment of a mouse skin model of the lichenification in human chronic eczematous dermatitis. Br J Dermatol 2007; 156(5): 884-91.

[14] Denda M, Fuziwara S, Inoue K. Influx of calcium and chloride ions into epidermal keratinocytes regulates exocytosis of epidermal lamellar bodies and skin permeability barrier homeostasis. J Invest Dermatol 2003; 121(2): 362-7.

[15] Kurzen H, Henrich C, Booken D, et al. Functional characterization of the epidermal cholinergic system in vitro. J Invest Dermatol 2006; 126(11): 2458-72.
[16] Stark HJ, Szabowski A, Fusenig NE, Maas-Szabowski N. Organotypic cocultures as skin equivalents: A complex and sophisticated in vitro system. Biol Proced Online 2004; 6: 55-60.

[17] Oliver MH, Harison NK, Bishop JE, Cole PJ, Laurent GJ. A rapid and convenient assay for counting cells cultured in microwell plates: application for assessment of growth factors. J Cell Sci 1989; 92: 513-518.

[18] Hoffjan S, Stemmler S. On the role of the epidermal differentiation complex in ichthyosis vulgaris, atopic dermatitis and psoriasis. $\mathrm{Br} \mathrm{J}$ Dermatol 2007; 157(3): 441-9.

[19] Proksch E, Folster-Holst R, Jensen JM. Skin barrier function, epidermal proliferation and differentiation in eczema. J Dermatol Sci 2006; 43(3): 159-69.

[20] Denda M, Inoue K, Inomata S, Denda S. Gamma-Aminobutyric acid (A) receptor agonists accelerate cutaneous barrier recovery and prevent epidermal hyperplasia induced by barrier disruption. Invest Dermatol 2002; 119(5): 1041-7.

[21] Fuziwara S, Inoue K, Denda M. NMDA-type glutamate receptor is associated with cutaneous barrier homeostasis. J Invest Dermato 2003; 120(6): 1023-9.

[22] Fuziwara S, Suzuki A, Inoue K, Denda M. Dopamine D2-like receptor agonists accelerate barrier repair and inhibit the epiderma hyperplasia induced by barrier disruption. J Invest Dermatol 2005; 125(4): 783-9.

[23] Langosch D, Thomas L, Betz H. Conserved quaternary structure of ligand-gated ion channels: the postsynaptic glycine receptor is a pentamer. Proc Natl Acad Sci USA 1988; 85(19): 7394-8.

[24] Cascio M. Structure and function of the glycine receptor and related nicotinicoid receptors. J Biol Chem 2004; 279(19): 19383-6.

[25] Haverkamp S, Müller U, Zeilhofer HU, Harvey RJ, Wässle H Diversity of glycine receptors in the mouse retina: localization of the alpha2 subunit. J Comp Neurol 2004; 477(4): 399-411.

[26] Neumann SB, Seitz R, Gorzella A, Heister A, Doeberitz MK, Becker CM. Relaxation of glycine receptor and onconeural gene transcription control in NRSF deficient small cell lung cancer cell lines. Brain Res Mol Brain Res 2004; 120(2): 173-81.

[27] Yadid G, Youdim MB, Zinder O. Uptake and receptor sites for glycine in isolated bovine adrenal medulla chromaffin cells. Neuroscience $1993 ; 55(4)$ : 1147-52

[28] Fischer M, William T, Helmbold P, Wohlrab J, Marsch WCh Expression of epidermal N-methyl-D-aspartate receptors (NMDAR1) depends on formation of the granular layer--analysis in diseases with parakeratotic cornification. Arch Dermatol Res 2004a; 296(4): 157-62

[29] Fischer M, Glanz D, William T, Klapperstuck T, Wohlrab J, Marsch WCh. N-methyl-D-aspartate receptors influence the intracellular calcium concentration of keratinocytes. Exp Dermatol 2004b; 13(8): 512-9. 